\title{
Effect of Primary Interference on Cognitive Relay Network
}

\author{
Nafise Sadeghi ${ }^{1}$, Rouhollah Aghajani ${ }^{1}$ \\ ${ }^{1}$ Department of Electrical Engineering, Najafabad Branch, Islamic Azad University, \\ Najafabad,Isfahan, Iran
}

\begin{abstract}
Cognitive relay network is a method for optimizing frequency spectrum utilization. What's important in these networks is to transmit data such that none of primary and secondary users cause destructive interference to other users. Although primary interference affect cognitive network performance, but is neglected in former researches. In this paper, we show cognitive network performance by calculating outage probability. We consider both primary and secondary interference links. Finally, our study is corroborated by representative numerical example. Simulation results demonstrate that increasing interference threshold increase outage probability and increasing data transmit rate cause outage probability increase.
\end{abstract}

Keywords: cognitive relay, cognitive radio, outage probability, signal to noise ratio. 


\section{Introduction}

$\mathrm{A}$ fter the great growth of wireless networks, using relay was proposed to improve system throughput. Using relay as a communication channel between transmitter and receiver was introduced in 1971 and its capacity lower band was calculated for a relay channel [1]. In 1988 capacity of a relay channel was determined without considering noise [2]. Then, capacity lower band and upper band was considered in 2005 [3].

Frequency spectrum is limited, so should be used optimum. Simon and his colleagues express advantages and constraints of wireless networks in [4] and present some solutions to resolve the constraints. Optimum use of frequency spectrum, needs changing traditional management of frequency spectrum to dynamic management. Simon emphasizes cooperative and cognitive networks.

The main idea of cooperative communication is that all users cooperate in sending signal to its destination [5]. User's information sends not only by itself, but also by other users. So received information will be more reliable and fading is decreased by multiple sending [6].

Cognitive radio was proposed in 1999 to optimize the frequency spectrum utilization [7]. The word "cognitive" refers to system knowledge about its environment. Cognitive radio is an intelligent communication system that recognizes its environment, identifies useless spectrum opportunities and adapts data transmission due to its environment changes, Haykin said [13]. In other word radio's parameters such as frequency, modulation power, band width is changed by changing environment features [6], [14]. Although there is different definition of cognitive radio, which all of them emphasis these features: observation, adaptation, intelligence and analysis.

Because using relay improve system throughput, combining cognitive radio and relay network play an important role in optimizing frequency spectrum utilization and system reliability [16], [17]. Cognitive relay network with AF and DF strategies was studied in 2004.
Author in [8] calculate outage probability of each strategy in regions with high SNR. Then a network with a single relay was considered to maximize the capacity [9]. A similar system was selected to minimize the power in 2005. In 2010 Alizadeh consider a network contains pair of transmitter and receiver with several relay terminals between them.

Data transmission in cognitive relay network is done via two links: 1- form secondary user to destination (direct link) 2- form secondary user to relay then from relay to destination (relay link).

In most former researches, such as [18], secondary user is supposed so far from destination that data transmit via direct link become weak and can be neglected. Articles [19][21] discussed about networks with several relays and present some techniques to select best relay for improving cognitive relay performance, then calculated system capacity and outage probability. Author in [22] study the effect of secondary users interference on primary user power control.

The effect of primary interference on secondary user almost is neglected in former researches. Purpose of this paper is to show cognitive relay network performance considering both primary and secondary interference.

The rest of this paper is recognized as follows: section 2 describes the system model. In section 3 outage probability is obtained to show cognitive relay network performance. Simulation results are given in section 4 . Finally, section 5 concludes this paper.

\section{SYSTEM MODEL}

The model is considered here contain several transmitters and a receiver, is named primary network. Besides primary network there is a pair of secondary transmitter and receiver. We use relay to improve channel reliability. This relay belongs to primary network before, but now it participates in secondary network data transmitting too. Relay use DF protocol, means that decode received signal then send it to destination. 


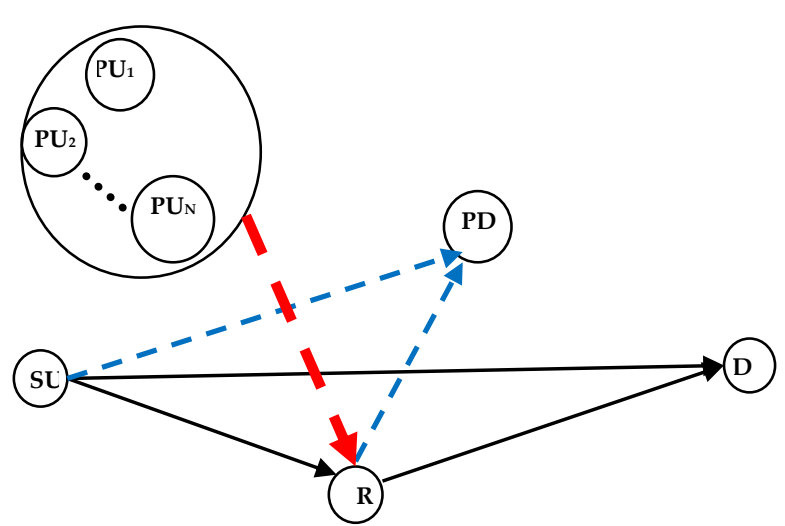

Figure 1: Cognitive relay network model.

As long as secondary user has no destructive interference on primary users, it can transmit data on primary network frequency band. As shown in fig. 1, there are two link between secondary user and its destination, direct link and relay link. Relay link has two phases, first phase is from secondary user to relay and second phase is from relay to destination. These transmission links are shown in black.

Blue link are shown secondary interference links that have destructive effect on primary network. Besides these interference links, there is a group of interference links that neglected in previous researches. This group named primary interference, is shown in red. We suppose primary users to secondary destination distance so long that the effect of primary users on secondary destination can be neglected. The quality of service is guaranteed when none of primary and secondary users have destructive interference on each other.

\section{OUTAGE PROBABILITY}

Outage occurs when data doesn't receive at destination correctly. Before calculate the outage probability, define some notations. $h_{P D, R}$ is channel coefficient of primary users to relay link. $h_{P D, D}$ is channel coefficient of primary users to secondary destination link. $\mathrm{h}_{\mathrm{SU}, \mathrm{R}}$ is channel coefficient of secondary users to relay link and $h_{R, D}$ is channel coefficient of relay to secondary destination link.

Primary users are supposed near each other. All channel coefficients are independent with normal distribution and noise is considered as AWGN with variance $\sigma_{\mathrm{N}}{ }^{2}$.

As said before secondary users connect to destination via direct and relay links, hence outage probability calculation is under the influence of both direct and relay links. This feature makes our solution as an optimum method.

When secondary user want transmit its information to destination, first send the normalized signal $\mathrm{x}_{\mathrm{n}}$ to relay $\mathrm{R}$ and destination D. Received signal at relay R and destination D can be written as (1) and (2) respectively:

$$
y_{R}=\sqrt{P_{n}} h_{S U, R} x_{n}+\sqrt{P_{p u}} h_{P U, R} x_{n}^{\prime}+n_{R}
$$

$y_{r}$ is received signal at relay, $P_{n}$ and $P_{p u}$ denote secondary user transmitted signal power and primary user transmitted signal power respectively. $\mathrm{n}_{\mathrm{R}}$ is the noise of relay.

$$
\begin{aligned}
& y_{D}^{(1)}=\sqrt{P_{n}} h_{S U, D} x_{n}+\sqrt{P_{p u}} h_{P U, D} x_{n}^{\prime}+n_{D}^{(1)}(2) \\
& \mathrm{y}_{\mathrm{D}}^{(1)} \text { denotes, received signal at secondary }
\end{aligned}
$$
destination and $n_{D}^{(1)}$ is the noise of destination channel.

Interference in primary destination should be less than a specific threshold, in this situation we have no destructive interference on them. So we have:

$$
P_{p u}=\frac{I_{t h}}{\max \left|h_{P U, D}\right|^{2}}
$$

$I_{t h}$ is the maximum interference that can be tolerated. We have for secondary user transmitted signal power:

$$
P_{n}=\frac{I_{t h}}{\left|h_{S U, P D}\right|^{2}}
$$

And similar previous:

$$
P_{R}=\frac{I_{t h}}{\left|h_{R, P D}\right|^{2}}
$$

In this way signal power is determined due to maximum tolerable interferences. After first phase, relay send decoded signal to secondary destination in second phase.

$$
\begin{aligned}
& y_{D}^{(2)}=\sqrt{P_{R}} h_{R, D} y_{R}+n_{D}^{(2)} \\
& \mathrm{n}_{\mathrm{D}}^{(2)} \text { is noise of destination in secondary phase. }
\end{aligned}
$$
Then signal to noise ratio is calculated as follow:

$$
S N R_{R}=\frac{I_{t h} \frac{\left|h_{S U, R}\right|^{2}}{\left|h_{S U, P D}\right|^{2}}}{\sigma_{N}{ }^{2}+P_{p u} \sum_{n=1}^{N}\left|h_{P U, R}\right|^{2}}
$$

$\mathrm{SNR}_{R}$ is signal to noise ratio at relay. The above equation clearly specifies that relay noise contain normal distributed channel noise and primary users interferences.

$$
S N R_{D}=\frac{{ }_{t h} \frac{\left|h_{S U, R}\right|^{2}}{\left|h_{S U, P D}\right|^{2}}}{\sigma_{N}{ }^{2}}
$$

$\mathrm{SNR}_{\mathrm{D}}$ is signal to noise ratio at secondary destination. Since primary users to secondary destination distance is supposed long enough, primary users interferences become zero.

By considering MRC, signal to noise ratio can be written as follow:

$$
S N R=\frac{I_{t h} \frac{\left|h_{S U, D}\right|^{2}}{\left|h_{S U, P D}\right|^{2}}+I_{t h} \frac{\left|h_{R, D}\right|^{2}}{\left|h_{P U, R}\right|^{2}}}{\sigma_{N}{ }^{2}}
$$

SNR denotes signal to noise ratio at secondary destination by MRC technique. Compute cumulative distribution function CDF 
to obtain outage probability of system. By knowing that hsu,R, hR,D , hsu,D , hsu,PD , hPU,R are normal distributed by variances $\alpha, \zeta, \beta, \varepsilon, \eta$ respectively, distribution functions of each SNRs can be obtained as:

$$
\begin{aligned}
& F_{X}(x)=1-\frac{A I_{t h}}{x P_{p u}} \times \frac{1}{(n-1) !} \times \frac{1}{\eta^{2 n}} \times B^{n-1} \times \\
& \exp \left(\frac{B}{\eta^{2}}\right) \times \Gamma(n) \Gamma\left(1-n, \frac{B}{\eta^{2}}\right)
\end{aligned}
$$

$F_{X}(x)$ is cumulative distribution function of SNRr. A and B are define as $A=\frac{\alpha^{2}}{\varepsilon^{2}}, B=$ $\frac{A I_{t h}}{x P_{p u}}+\frac{\sigma_{N}^{2}}{P_{p u}}$.

Equation (10) is obtained by combining some normal distributed functions and need precise statistics information. In this way other distribution functions are obtained.

$$
F_{Y}(y)=\frac{y}{y+C}
$$

$F_{Y}(y)$ is cumulative distribution function of SNRD and $C$ defines as $C=\frac{\beta^{2} I_{t h}}{\varepsilon^{2} \sigma_{N}{ }^{2}}$. As we can see in equation (8), this function obtain from distribution of dividing two variable with specified distribution.

Cumulative distribution function of SNR is obtained as bellow:

$$
\begin{aligned}
& F_{U}(u)= \\
& \frac{D E \ln \left(\frac{D E}{\left(D+\frac{u \sigma_{N}^{2}}{I_{t h}}\right)\left(E+\frac{u \sigma_{N}^{2}}{I_{t h}}\right)}\right)+(D+E) \frac{u \sigma_{N}^{2}}{I_{t h}}+\left(\frac{u \sigma_{N}{ }^{2}}{I_{t h}}\right)^{2}}{\left(D+E+\frac{u \sigma_{N}{ }^{2}}{I_{t h}}\right)^{2}}
\end{aligned}
$$

In the above equation $=\frac{\beta^{2}}{\varepsilon^{2}}, E=\frac{\zeta^{2}}{\eta^{2}}$.

System outage probability is the probability that system wouldn't be able to transmit data correctly. Outage occurs when: 1)relay and destination don't receive data correctly 2) relay receive data correctly but finally destination fail to receive data from relay. It means:

$$
P_{\text {out }}=\left[\operatorname { P r } ( S N R _ { R } < \gamma _ { \text { th } } ) \& \operatorname { P r } \left(S N R_{D}<\right.\right.
$$$$
\left.\left.\gamma_{t h}\right)\right] \mid\left[\left(1-\operatorname{Pr}\left(S N R_{R}<\gamma_{t h}\right)\right) \& \operatorname{Pr}\left(S N R<\gamma_{t h}\right)\right]
$$

$\gamma_{\text {th }}$ is a determined threshold of signal to noise ratio. When signal to noise ratio is bigger than $\gamma_{\text {th }}$, means that data receive correctly at destination. Equivalently can write:

$$
P_{\text {out }}=\left[F_{X}\left(\gamma_{t h}\right) \& F_{Y}\left(\gamma_{t h}\right)\right] \mid[(1-
$$

$$
\left.\left.F_{X}\left(\gamma_{t h}\right)\right) \& F_{U}\left(\gamma_{t h}\right)\right]
$$

In the above equation " $\&$ " denoted logical operator AND, " | " denoted logical operator OR.

\section{SIMULATION}

In this section, model our cognitive relay network in Matlab to illustrate system operation. We choose outage probability as a factor to show how system performs. This probability was calculated after repeating the random experiment for $10^{8}$ times and averaging results.

Fig. 2 illustrates the outage probability for different maximum interference thresholds $(-5,0$, $5,10 \mathrm{~dB})$. According simulation results, system outage probability decreases when Ith increases. Red curve represents analytical results and black curve represents theoretical results. As seen in this diagram, analytical and theoretical results are near each other.

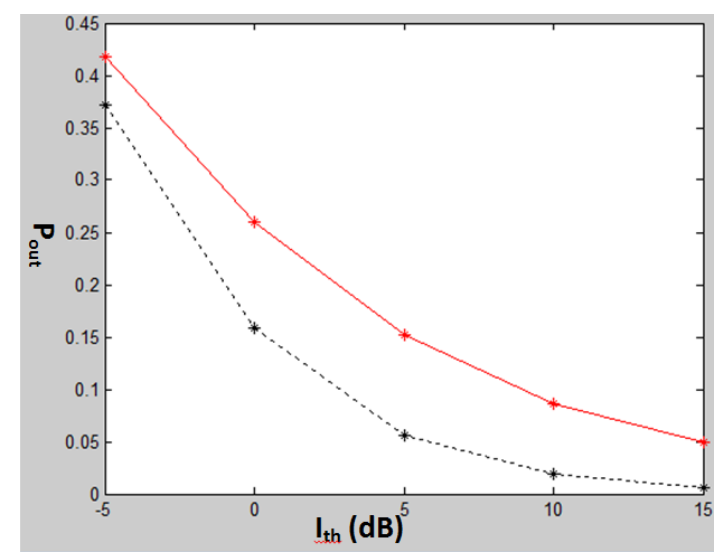

Figure 2: Outage probability in terms of interference threshold curve.

Fig.3 shows data transmit rate effect as another parameter that changes outage probability. It is natural the increase of data transmit rate increases the probability of failing data at destination so increases outage probability.

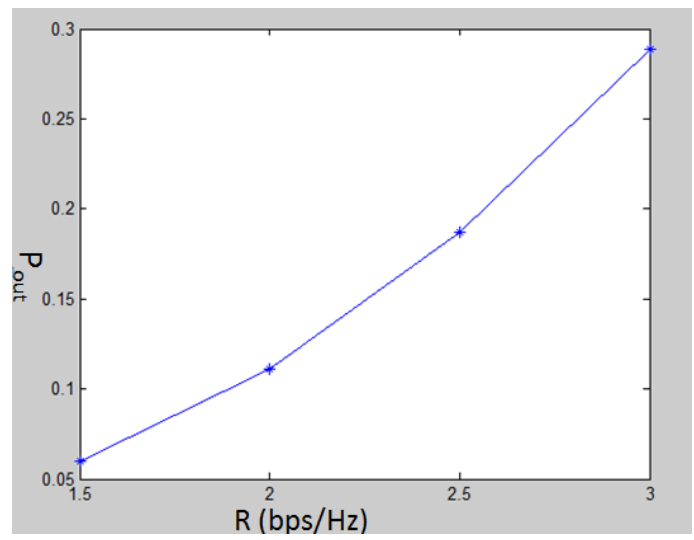

Figure 3: Outage probability in terms of data transmit rate curve 


\section{Conclusion}

In this paper both direct and relay links are considered in outage probability calculation, and this makes our method become optimum. Interference decreases probability of receiving data correctly. So obviously, considering the effect of primary users interferences on secondary user, increase outage probability.

In this paper, the effect of maximum interference threshold and data transmit rate on outage probability is shown. So that, the increase of maximum interference threshold decrease outage probability and the increase of data transmit rate results in outage probability increase.

To continue this research can consider more than one secondary user. Although this makes equations more complex but presents more accurate results.

\section{References}

Van Der Meulen, E. (1971). Three-terminal communication channels. Advances in Applied Probability, vol. 3, no. 1, pp. 120-154.

Zhang, Z. (1988). Partial converse for a relay channel. Information Theory, IEEE Transactions on vol. 34, no. 5, pp.1106-1110.

Anders Høst-Madsen and Junshan Zhang. (2005). Capacity Bounds and Power Allocation for Wireless Relay Channels. Information Theory, IEEE Transactions on vol. 51, no. 6, pp. 2020-2040.

Simeone, O., Gambini, J., Bar-Ness, Y. and Spagnolini, U. (2007). Cooperation and Cognitive Radio Communications, 2007. ICC'07. IEEE International Conference on. IEEE. Jun. 2007.

Weifeng, Su., Sadek K.A. and Ray Liu K.J. (2008). Cooperative communication protocols in wireless networks: Performance analysis and optimum power allocation. Wireless Personal Communications. vol. 44, no. 2, pp. 181-217.

Andrea Goldsmith. (2005). Wireless communications. Cambridge University Press.

Mitola, J. III. (2000). Cognitive radio: An integrated agent architecture for software defined radio. Ph.D. dissertation, Royal Inst. Of Technol., Stockholm, Sweden, 2000.

$\mathrm{Hu}, \mathrm{D}$. and Mao, S. (2010). Cooperative relay in cognitive radio networks: Decode-andforward or amplify-and-forward?, Global Telecommunications

Conference (GLOBECOM 2010).

Deng, X. and Haimovich, A. (2005). Power allocation for cooperative relaying in wireless networks. IEEE Communications Letters, vol.9, no. 11, pp.994-996.

Jingmei, Z., Qi, Z., Chunju, S., Ying, W., Ping, Z. and Zhang, Z. (2004). Adaptive optimal transmit power allocation for two-hop nonregenerative wireless relaying system. Vehicular Technology Conference, 2004. VTC 2004-Spring. 2004 IEEE 59th. Vol. 2. IEEE.

Ardalan alizadeh, Seyed mohammad-sajad Sadough. (2010). Power Minimization in Unidirectional Relay Networks with Cognitive Radio Capabilities. Telecommunications (IST), 2010 5th International Symposium on. IEEE.

Khuong Ho-Van, Vo Nguyen Quoc Bao. (2013). On The Performance of Underlay Relay Cognitive Networks. REV Journal on Electronics and Communications, vol. 3, no. $1-2$.

Stefano Rini, Daniela Tuninetti, Natasha Devroye, and Andrea J. Goldsmith. (2014). On the Capacity of the Interference Channel With a Cognitive Relay. Information Theory, IEEE Transactions on vol. 60, no.4, pp. 21482179.

Renani, R.A., Saadat, R., Aref, M.R., Mirjalily, G. (2011). SER of M-PSK modulation in ncremental selective decode-and-forward cooperative communications over Rayleigh fading channels. Advanced Communication Technology (ICACT), 2011 13th International Conference on, Seoul, pp. 432-437.

Lisheng Fan, Xianfu Lei, Trung Q. Duong, Rose Qingyang $\mathrm{Hu}$, and Maged Elkashlan. (2014). Multiuser Cognitive Relay Networks: Joint Impact of Direct and Relay Communications. Wireless Communications, IEEE Transactions on vol. 13, no. 9, pp. 5043-5055. 
Wenjing Yue, Baoyu Zheng, Qingmin Meng. (2009). Optimal Power Allocation for Cognitive Relay Networks, Wireless Communications \& Signal Processing, 2009. WCSP 2009. International Conference on. IEEE.

Yonghui Li, Vucetic, B., Zhendong Zhou, Dohler, M. (2007). Distributed Adaptive Power Allocation for Wireless Relay Networks. Wireless Communications, IEEE Transactions on vol. 6, no. 3, pp. 948-958.

Guo, Y., Kang, G., Zhang, N., Zhou, W. and Zhang, P. (2010). Outage Performance of Relay-Assisted Cognitive-Radio System under, Spectrum-Sharing Constraints. Electron. Lett., vol. 46, no. 2, pp. 182-184.

Sagong, S., Lee, J. and Hong, D. (2011). Capacity of Reactive DF Scheme in Cognitive Relay Networks. Wireless Communications, IEEE Transactions on vol. 10, no. 10, pp. 1536-1276.

Lee, J., Wang, H., Andrews, J. G. and Hong, D. (2011). Outage Probability of Cognitive Relay Networks with Interference Constraints, Wireless Communications, IEEE Transactions on vol. 10, no. 2, pp. 390-395.

Guimaraes, F.R.V., Costa , D.B, Tsiftsis, T.A., Cavalcante, C.C and karagiannidis, G.K. (2014). Multiuser and Multirelay Cognitive Radio Networks Under Spectrum-Sharing Constraints, IEEE Transactions Vehicular Technology, vol. 63, no. 1, pp. 433-439.

Khan Sohaib, Yonghoon Choi and Youngnam Han. (2010). Outage Improvement in Cognitive Relay Networks by Using a Generalized Regional Model, Vehicular Technology Conference Fall (VTC 2010-Fall) IEEE 72nd, pp. 1-5.

Ekram Hossain,Vijay Bhargava. (2007). Cognitive Wireless Communication Networks, springer.

Akaike, H. (1973). Information theory and an extension of the maximum likelihood principle. Em: Proceedings of the 2nd International Symposium on Information Theory, pp. 267-281.

Ferrari, S. L. P., Cribari-Neto, F. (2004). Beta regression for modelling rates and proportions. Journal of Applied Statistics, 31(7), 799-815.
McCullagh, P., Nelder, J. (1989). Generalized linear models, 2o edn. Chapman and Hall.

R Development Core Team (2009). R: A language and environment for statistical computing. $\mathrm{R}$ Foundation for Statistical Computing, Vienna, Austria, URL 